\title{
Primary splenic B-cell lymphoma, unclassifiable, with features intermediate between diffuse large B-cell lymphoma and classical Hodgkin lymphoma: A case report
}

\author{
FENG SHI $^{1}$, QUAN ZHOU ${ }^{1}$, YING GAO $^{1}$, XIANG-QING CUI $^{2}$ and HONG CHANG ${ }^{1}$ \\ ${ }^{1}$ Department of Pathology, Beijing Shijitan Hospital, Capital Medical University, \\ Beijing 100038; ${ }^{2}$ Department of Pathology, Pu Ren Hospital, Beijing 100062, P.R. China
}

Received March 27, 2015; Accepted April 15, 2016

DOI: $10.3892 / \mathrm{ol} .2016 .4840$

\begin{abstract}
B-cell lymphoma (BCL), unclassifiable, with features intermediate between diffuse large BCL (DLBCL) and classical Hodgkin's lymphoma (CHL), is a novel entity to the World Health Organization classification system. These tumors are rare aggressive lymphomas that have a poor prognosis. The present study reports the case of a patient with one such lymphoma that occurred in the spleen, which expressed cluster of differentiation (CD)20, CD79 $\alpha$, melanoma associated antigen (mutated) 1, BCL6, CD15 and CD30. Polymerase chain reaction analysis demonstrated a clonal rearrangement of the genes coding for immunoglobulin heavy chains. The tumor cells demonstrated a negative reaction in the Epstein-Barr virus-encoded small RNA assay. Following the diagnosis of unclassifiable BCL, with intermediate features between DLBCL and CHL, the patient received 7 cycles of the $\mathrm{CHOP}$ regimen, and so far, has been in good general condition and tumor-free for 17 months.
\end{abstract}

\section{Introduction}

B-cell lymphoma (BCL), unclassifiable, with features intermediate between diffuse large BCL (DLBCL) and classical Hodgkin's lymphoma (CHL), also known as 'large BCL (LBCL) with Hodgkin features' and 'Hodgkin-like anaplastic large cell lymphoma' (HD-like ALCL), is a borderline lymphoma with features of LBCL and CHL (1). These tumors are rare aggressive lymphomas, often associated with mediastinal disease, and similar cases have reported the peripheral lymph node groups as a primary site (1). Occasionally, these lymphomas may spread to the lung, liver, spleen and bone marrow. These

Correspondence to: Dr Hong Chang, Department of Pathology, Beijing Shijitan Hospital, Capital Medical University, 10 Tieyi Road, Haidian, Beijing 100038, P.R. China

E-mail: cherryshifeng@163.com

Key words: B-cell lymphoma, spleen, unclassifiable, gray zone, lymphoma lymphoma are most common in young men, and the majority of cases have been reported from Western countries, being less common in African-American and Asian populations. No consensus exists regarding the optimum treatment for this tumor. The present study reports the case of patient with one such lymphoma that occurred in the spleen.

\section{Case report}

A 48-year-old female Chinese patient presented to the Department of Pathology of Beijing Shijitan Hospital (Beijing, China) on May 2013 with light pain in the upper left abdomen that had lasted for 1 year. The pain did not radiate to the shoulders and back and the pain worsened when the patient was tired or changed position. The patient demonstrated no B symptoms, including fever, night sweats or weight loss. On investigation, the complete blood count (CBC) and the bone marrow biopsy of the patient were normal, and the liver and renal functions were within the normal range. The ultrasound and computed tomography (CT) scans showed splenomegaly without hepatomegaly, and a focal mass in the spleen. A CT scan revealed movable enlarged lymph nodes in left neck and bilateral inguinal folds, which measured 3 and $4 \mathrm{~cm}$ in diameter, respectively, and were not accompanied by pain or fever. The patient had previously received a uterectomy due to the presence of a uterine leiomyoma half a year prior to presentation.

The patient underwent a full splenectomy for diagnosis on May 2013. Intra-operatively, the spleen was found to be enlarged and congested, and one polar adhered to pancreas. Macroscopically, the spleen measured $14.5 \times 11.5 \times 8.5 \mathrm{~cm}$ and was $602 \mathrm{~g}$ in mass. A solitary mass with scalloped margins and a grayish-white color was located at one end of the spleen and involved the splenic capsule.

The surgical specimen was fixed by $4 \%$ neutral formaldehyde, and subjected to paraffin embedding, conventional sectioning and hematoxylin and eosin staining. The histopathological sections revealed that the tumor was composed of large atypical pleomorphic lymphoid cells with prominent nuclei and open chromatin, and collagen bands (Fig. 1A). Lacunar, Hodgkin and multinuclear giant cells were also present among large atypical cells, scattered lymphocytes, eosinophilic granulocytes, neutrophile granulocytes and 
Table I. Antibodies used for immunohistochemistry.

\begin{tabular}{lll}
\hline Antibody target antigen & \multicolumn{1}{c}{ Characteristics } & Catalogue number \\
\hline CD3 & Polyclonal rabbit anti-human & IR503 \\
CD20 & Monoclonal mouse anti-human & IR604 Clone L26 \\
CD30 & Monoclonal mouse anti-human & IR602 Clone Ber-H2 \\
CD15 & Monoclonal mouse anti-human & IR062 Clone Carb-3 \\
CD10 & Monoclonal mouse anti-human & IR648 Clone 56C6 \\
CD79a & Monoclonal mouse anti-human & IR621 Clone JCB117 \\
CD45RO & Monoclonal mouse anti-human & IR751 Clone 2B11+PD7/26 \\
BCL2 & Monoclonal mouse anti-human & IR614 Clone 124 \\
BCL6 & Monoclonal mouse anti-human & ir625 clone pg-b6p \\
ALK & Monoclonal Rabbit Anti-Human & CD246 \\
\hline
\end{tabular}

$\mathrm{CD}$, cluster of differentiation; BCL, B-cell lymphoma; ALK, anaplastic lymphoma kinase.
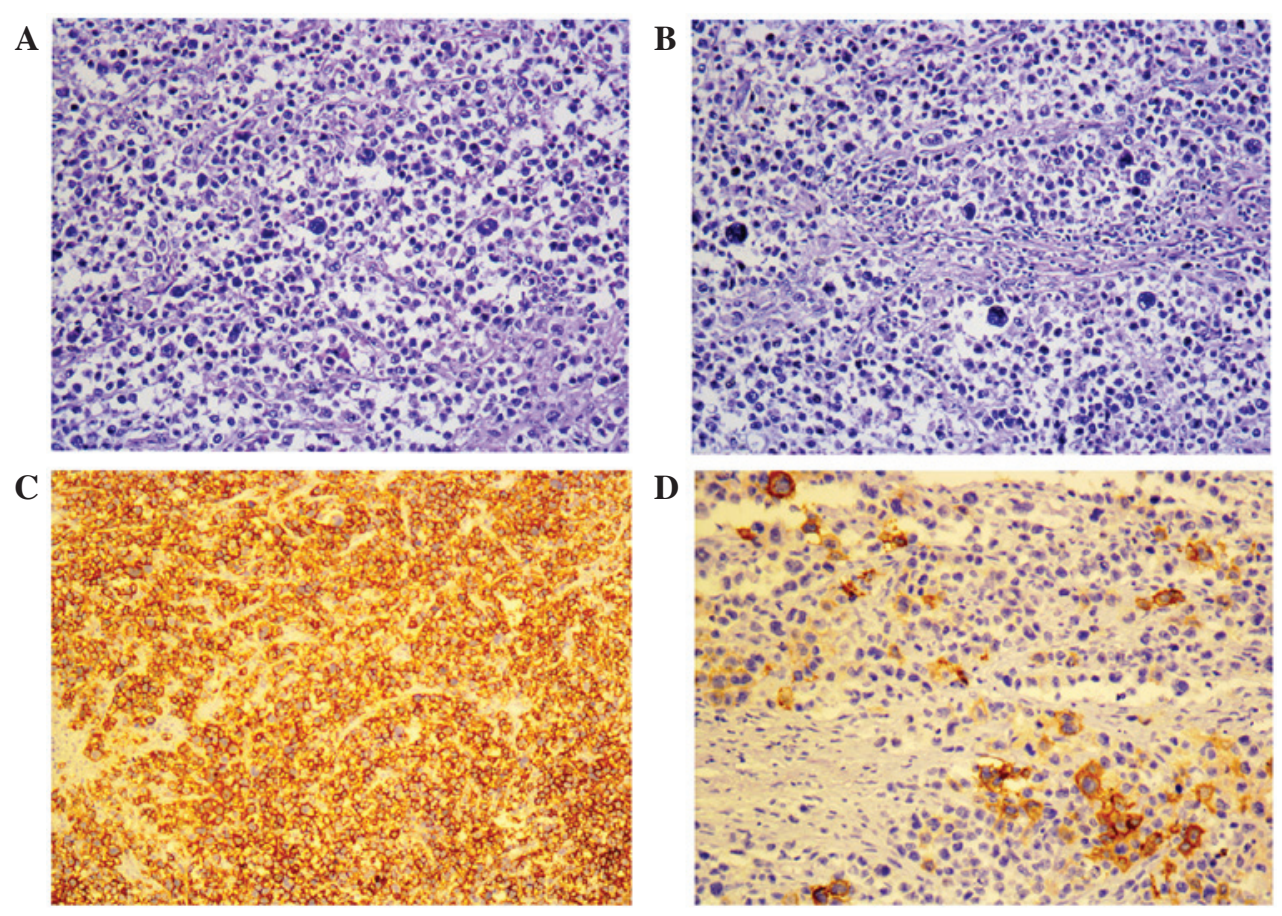

Figure 1. (A) Pleomorphic lymphoid cells and collagen bands (H\&E staining; magnification, x100). (B) Pleomorphic large lymphoid cells with prominent nuclei and open chromatin (H\&E staining; magnification, x200). The tumor expressed (C) CD20 (IHC; magnification, x100) and (D) CD30 (IHC; magnification, x200). CD, cluster of differentiation; H\&E, hematoxylin and eosin staining; IHC, immunohistochemistry.

histocytes (Fig. 1B). Immunohistochemical (IHC) examination was performed using the EnVision method with FLEX Ready-to-Use and Autostainer Link Solution for IHC (Dako, Glostrup, Denmark). Table I contains detailed information about the antibodies used for the analysis, which were purchase from Dako. By IHC, the large atypical lymphoid, Hodgkin and multinuclear giant cells were positive for cluster of differentiation (CD)20, CD79 $\alpha$, melanoma associated antigen (mutated) 1 (MUM1), BCL6, CD15 and CD30 (Fig. 1C-F), but negative for CD3, CD10, anaplastic lymphoma receptor tyrosine kinase (ALK) and CD45RO. These tumor cells demonstrated a negative reaction in the Epstein-Barr virus (EBV)-encoded small RNA assay (EBV kit; catalogue number ISH-5021; OriGene Technologies,
Inc., Rockville, MD, USA). Polymerase chain reaction (PCR) analysis was conducted with IdentiClone ${ }^{\mathrm{TM}}$ IGH Gene Clonality Assay (catalogue number 9-101-0020; Invivoscribe Technologies, Inc., San Diego, CA, USA) under the following cycling conditions: Denaturation at $95^{\circ} \mathrm{C}$ for $7 \mathrm{~min}$, followed by 35 cycles of $95^{\circ} \mathrm{C}$ for $45 \mathrm{sec}, 60^{\circ} \mathrm{C}$ for $45 \mathrm{sec}$ and $72^{\circ} \mathrm{C}$ for $90 \mathrm{sec}$ and a final extension at $72^{\circ} \mathrm{C}$ for $10 \mathrm{~min}$. The PCR results demonstrated a clonal rearrangement of the genes coding for immunoglobulin (Ig) heavy chains.

Based on these findings, the patient was diagnosed with splenic LBCL, with features between DLBCL and CHL. Following diagnosis, the patient received 7 cycles of the CHOP regimen, consisting of $1 \mathrm{~g}$ cyclophosphamide (day 1 ), $80 \mathrm{mg}$ pirarubicin (day 1), $4 \mathrm{mg}$ vindesine (day 1) and $15 \mathrm{mg}$ 
dexamethasone (days 1-5), and she has been in good general condition and tumor-free for 17 months.

The patient, who is currently under follow-up, provided written informed consent for the publication of the present case report.

\section{Discussion}

Common lymphomas that have been reported in the spleen include DLBCL, marginal zone BCL, Hodgkin's lymphoma, follicular lymphoma and the rare type of lymphoma reported in the present study (2-7). The current classification of lymphoid neoplasms is based on clinical information, morphology, immunophenotype and molecular genetic characteristics (1-6). Despite technical and scientific progress, certain aggressive BCLs with features that overlap two types of lymphoma remain a challenge to classify. The updated 2008 World Health Organization classification of Tumours of the Hematopoietic and Lymphoid Tissues has addressed this problem by creating two novel provisional categories of BCLs: Unclassifiable, i) with features intermediate between DLBCL and CHL; or ii) with features intermediate between DLBCL and Burkitt's lymphoma (1). The former is the lymphoma reported in the present study, and is a B lineage lymphoma that demonstrates overlapping clinical, morphological and immunophenotypical features, between CHL and DLBCL. These lymphomas are most common in young men, and the majority of cases reported have been from Western countries (1). These are rare aggressive lymphomas that are often associated with mediastinal disease; similar cases have been reported that show the peripheral lymph node groups as a primary site, and the tumors are rarely found in the spleen $(6,8,9)$. The present patient is a 48-year-old Chinese woman and the lymphoma occurred in spleen.

The present patient had no severe complications and underwent a splenectomy. Pathology showed that the lymphoma was composed of a confluent, sheet-like growth of pleomorphic tumor cells in a diffusely fibrotic stroma. The cells were larger and more pleomorphic compared with typical cases of DLBCL, and a few centroblast-like cells were present. Pleomorphic cells resembling lacunar cells and Hodgkin cells comprised the majority of the infiltrate. Scattered eosinophils, lymphocytes and histocytes were present and the necrotic regions did not contain neutrophilic infiltrates. According to IHC, the lymphoma cells expressed CD45, CD20, CD79a, MUM1, Bc16, CD30 and CD15, but not CD3, CD10, ALK or CD45RO. These tumor cells can often be found using EBV-encoded small RNA assays and PCR analysis, which demonstrate clonal rearrangements of the genes coding for Ig heavy chains (8-10). No EBV infection was identified in the present case. BCL, unclassifiable, with features intermediate between DLBCL and CHL have a differential diagnosis compared with with DLBCL, CHL and anaplastic large cell lymphoma.

BCL, unclassifiable, with features intermediate between DLBCL and CHL generally have a more aggressive clinical course and a poorer outcome compared with $\mathrm{CHL}$ and DLBCL $(6,9)$. No consensus exists regarding the optimum treatment for the tumor; however, certain studies propose that therapy for an aggressive LBCL has been effective, while others propose the use of a Hodgkin-type regimen (9,11-15). Certain studies suggest that the transitional lymphoma is relatively resistant to Hodgkin-based chemotherapy (9). Wang et al (16) reported 16 cases of this type of lymphoma, the majority of which appeared to be insensitive to immunochemotherapy (CHOP regimen), and only 4/13 cases had partial response. Of these, 2 cases received radiotherapy subsequent to chemotherapy and 2 cases were subjected to autologous stem cell transplantation. In the present case report, the patient received 7 cycles of the $\mathrm{CHOP}$ regimen and has been in a good general condition and tumor-free for 17 months following the splenectomy.

In conclusion, the present study reports a case of primary splenic BCL, unclassifiable, with features intermediate between DLBCL and HCL, which occurred in a 48-year-old Chinese woman. This lymphoma is rarely found to occur in the spleen. The patient has received 7 cycles of the CHOP regimen and has been tumor-free for 17 months. The lymphoma is rare and the most appropriate treatment remains controversial, hence additional studies are required to analyze the best treatment plan.

\section{References}

1. Swerdlow SH, Campo E, Harris NL, Jaffe ES, Pileri SA, Stein H, Thiele J and Vardiman JW (eds): WHO classification of tumours. In: WHO Classification of Tumours of Haematopoietic and Lymphoid Tissues. Vol 2. 4th edition. IARC Press, Lyon, pp267-268, 2008.

2. Iriyama N, Horikoshi A, Hatta Y, Kobayashi Y, Sawada S and Takeuchi J: Localized, splenic, diffuse large B-cell lymphoma presenting with hypersplenism: Risk and benefit of splenectomy. Inter Med 49: 1027-1030, 2010.

3. Kurup SK, Levy-Clarke G, Calvo KR, Jaffe ES, Nussenblatt RB and Chan CC: Primary diffuse large B-cell lymphoma of the spleen with coincident serous retinal detachments responsive to corticosteroids. Clin Experiment Ophthalmol 35: 468-472, 2007.

4. Ding YL and Wang SY: Gastrosplenic fistula due to splenic large B-cell lymphoma. J Res Med Sci 17: 805-807, 2012.

5. Matutes E: Splenic marginal zone lymphoma: Disease features and management. Expert Rev Hematol 6: 735-745, 2013.

6. Asano N, Suzuki R, Matsuo K, Kagami Y, Ishida F, Tamaru JI, Jin GS, Sato Y, Shimoyama Y and Yoshino T, et al: Cytotoxic molecule expression is predictive of prognosis in Hodgkin's-like anaplastic large cell lymphoma. Histopathology 50: 705-715, 2007.

7. Kim JK, Hahn JS, Kim GE and Yang WI: Three cases of diffuse large B-cell lymphoma presenting as primary splenic lymphoma. Yonsei Med J 46: 703-709, 2005.

8. Gardner RV, Velez MC, Ode DL, Lee JW and Correa H: Gamma/delta T-cell lymphoma as a recurrent complication after transplantation. Leuk Lymphoma 45: 2355-2359, 2004.

9. Travers-Glehen A, Pittaluga S, Gaulard P, Sorbara L, Alonso MA, Raffeld M and Jaffe ES: Mediastinal gray zone lymphoma: The missing link between classic Hodgkin's lymphoma and mediastinal large B-cell lymphoma. Am J Surg Pathol 29: 1411-1421, 2005.

10. Rüdiger T, Jaffe ES, Delsol G, deWolf-Peeters C, Gascoyne RD, Georgii A, Harris NL, Kadin ME, MacLennan KA, Poppema S, et al: Workshop report on Hodgkin's disease and related diseases ('gray zone' lymphoma). Ann Oncol 9 (Suppl 5): S31-S38, 1998.

11. Portlock CS, Donnelly GB, Qin J, Straus D, Yahalom J, Zelenetz A, Noy A, O'Connor O, Horwitz S, Moskowitz C and Filippa DA: Adverse prognostic significance of CD20 positive Reed-Sternberg cells in classical Hodgkin's disease. Br J Haematol 125: 701-708, 2004.

12. Tzankov A, Krugmann J, Fend F, Fischhofer M, Greil R and Dirnhofer S: Prognostic significance of CD20 expression in classical Hodgkin lymphoma: A clinicopanthological study of 119 cases. Clin Cancer Res 9: 1381-1386, 2003. 
13. Grant C, Dunleavy K, Eberle FC, Pittaluga S, Wilson WH and Jaffe ES: Primary mediastinal large B-cell lymphoma, classic Hodgkin lymphoma presenting in the mediastinum, and mediastinal gray zone lymphoma: What is the oncologist to do? Curr Hematol Malig Rep 6: 157-163, 2011.

14. Dunleavy K, Grant C, Eberle FC, Pittaluga S, Jaffe ES and Wilson WH: Gray zone lymphoma: Better treated like Hodgkin lymphoma or mediastinal large B-cell lymphoma? Curr Hematol Malig Rep 7: 241-247, 2012.
15. Parikh JG, Strom T and Stone I: MYC negative rectal B-cell lymphoma, unclassifiable, with features intermediate between diffuse large B-cell lymphoma and Burkitt's lymphoma in an immunocompetent patient. Case Rep Pathol 2013: 302304, 2013.

16. Wang L, Wang W, Li X, Guo Y and Zhu X: Grey zone lymphoma with features intermediate between diffuse large B-cell lymphoma and classical Hodgkin lymphoma: Clinicopathologic characterization of 16 cases showing different patterns. Zhonghua Bing Li Xue Za Zhi 43: 307-312, 2014 (In Chinese). 